\title{
THE SINGULARITIES OF THE PARAMETER SURFACE OF A MINIMAL ELLIPTIC THREEFOLD
}

\author{
Antonella Grassi \\ Tufts University
}

Let $\pi: X \rightarrow S$ be an elliptic fibration between smooth varieties. If $\operatorname{dim}(X)=2$, then $X$ can be assumed minimal and $K_{X}$ can be expressed as the pullback of a divisor from $S$. Moreover $S$ cannot be changed birationally. These observations were the first step in Kodaira's analysis of singular fibers [Kd]. In higher dimensions the birational geometry of the total space is more complicated and the parameter space can be modified birationally.

If $X$ has dimension 3 and is not uniruled, then $X$ has a minimal model (with terminal singularities) [Mo]. In earlier work [G1] we have shown that there exists a birationally equivalent elliptic fibration $\bar{\pi}: \bar{X} \rightarrow \bar{S}$ such that $\bar{X}$ is minimal and $K_{\bar{X}} \equiv \bar{\pi}^{*}\left(K_{\bar{S}}+\bar{\Lambda}\right)(\S 0)$, [G1]. Moreover $\bar{S}$ has at worst quotient singularities; it is not difficult to find examples where $\bar{S}$ is actually singular.

In this paper we study the singularities of this parameter surface $\bar{S}$. Although $\bar{S}$ is not uniquely determined by the birational equivalence class of the fibration, any two such $\bar{S}$ 's are related by a particular kind of birational map (0.4).

The following result covers the case of no multiple fibers.

Theorem 4.5. Let $\pi: X \rightarrow S$ and $\bar{S}$ be as above and assume that $\pi$ does not have multiple fibers outside a set of codimension 2. Then the only exceptional graphs of the minimal resolution of $\bar{S}$ are Hirzebruch-Jung strings of type $<n, q>, n \leq 6$.

In particular all the possible dual graphs are represented by the full circles in Tables 2, 3, 4.

In $\S 3$ we present examples of all these $<n, q>$ other than $n=5, q=2,3,4$.

If we allow multiple fibers things are more complicated.

Kawamata [Ka5] has shown that any quotient singularity can occur on $\bar{S}$. In his examples $\bar{S}$ has only one singular point $p, \bar{\pi}^{-1}(p)$ is a multiple fiber, and the elliptic fibration is isotrivial outside $p$. Moreover Dolgachev has shown me an example where $\bar{S}$ has a singular point $p, \bar{\pi}^{-1}(p)$ is a multiple fiber, and the elliptic fibration is not isotrivial outside $p$. However also in this case the only divisors of singular fibres passing through $p$ are the ones of multiple fibers.

I would like to thank J. Kollár and D. Morrison for offering valuable suggestions 
The following corollary is a consequence of Theorem 4.5.

We start with any elliptic fibration $X_{0} \rightarrow S_{0}$ and take a suitable birational equivalent fibration $X \rightarrow S$ of smooth varieties (see 0.3 ). Then the birational map $\phi: S \rightarrow \bar{S}$ is actually a morphism.

Corollary 4.7. Assume that all the exceptional curves for $\phi$ which are image of multiple fiber divisors have zero intersection with curves image of non multiple singular divisor. Then:

$$
\chi\left(\mathcal{O}_{X}\right)=-\frac{1}{2}\left\{\left[K_{\bar{S}}+\phi_{*} \pi_{*}\left(K_{X / S}\right)\right] \cdot \phi_{*} \pi\left(K_{X / S}\right)-\sum_{p \in V}\left(1-1 / n_{p}\right)\right\},
$$

where $V=\left\{p \in U\right.$ such that the fiber over the general point of $\phi^{-1}(p)$ is not multiple $\}$.

$\phi_{*} \pi_{*}\left(K_{X / S}\right)$ does not depend on the original choice of the birational model.

This formula generalizes to the 3-dimensional case the formula for the Euler characteristic for an elliptic surface $p: S \rightarrow C$, namely: $\chi\left(\mathcal{O}_{S}\right)=\operatorname{deg} p_{*} K_{S / C}$. If the fibration does not have multiple fiber, then $\chi\left(\mathcal{O}_{X}\right)=-\frac{1}{2}\left\{\left(K_{\bar{S}}+\bar{\Lambda}\right) \cdot \bar{\Lambda}-\right.$ $\left.\sum_{p \in V}\left(1-1 / n_{p}\right)\right\}$.

For example if the Kodaira dimension of $X$ is equal to 0 , then $\chi\left(\mathcal{O}_{X}\right)=1 / 2 \sum_{p \in V}(1-$ $\left.1 / n_{p}\right) .\left(K_{\bar{X}} \equiv \bar{\pi}^{*}\left(K_{\bar{S}}+\bar{\Lambda}\right)\right.$, see $\S 0$.) Other applications of this results can be found in $[\mathrm{G} 1]$

The hypothesis of the above Corollary is always satisfied if $\pi$ does not have multiple fibers outside a set of codimension 2 .

\section{Background results, notations and first properties.}

\section{Background.}

Let $\pi: X \rightarrow S$ be an elliptic fibration between smooth varieties.

Definition. Set:

$\Sigma_{X / S}=\{P \in S$ such that $\pi$ is not smooth over $P\}$

(the ramification divisor of $\pi$ )

$\Sigma_{X / S}^{o m}=\left\{P \in \Sigma_{X / S}\right.$ such that $\pi^{-1}(P)$ is a multiple fiber $\}$ and $\Sigma_{X / S}^{m}$ the closure of $\Sigma_{X / S}^{o m}$.

We write $\Sigma\left(\right.$ resp. $\left.\quad \Sigma^{m}\right)$ for $\Sigma_{X / S}\left(\operatorname{resp} \Sigma_{X / S}^{m}\right)$ when the fibration is well understood.

We denote by $J_{\infty}$ the divisor of poles of the elliptic modular function $\mathbf{J}$.

The following theorems hold:

Theorem 0.1 [F2]. Let $\pi: X \rightarrow S$ be an elliptic fibration between smooth varieties. Assuming that the modular function $\mathbf{J}$ extends to an holomorphic map $S \rightarrow \mathbb{P}^{1}$, then

$$
m K_{X}=\pi^{*}\left(m K_{S}+m \pi_{*}\left(K_{X / S}\right)+m \sum\left(\frac{m_{i}-1}{m_{i}} Y_{i}\right)\right)+m E-m G
$$

where the fiber over the general point of $Y_{i}$ is a multiple fiber of multiplicity $m_{i}, m$ is a multiple of the $\left\{m_{i}\right\}$ 's; $m E$ and $m G$ are effective divisors. 
Theorem 0.2 [Ka2]. Let $\pi: X \rightarrow S$ be an elliptic fibration between smooth varieties. Suppose that the ramification locus is a divisor with simple normal crossings. Then the modular function $\mathbf{J}$ extend to a holomorphic map $S \rightarrow \mathbb{P}^{1}, 12 \pi_{*}\left(K_{X / S}\right)$ is an invertible sheaf and $12 \pi_{*}\left(K_{X / S}\right) \cong \mathcal{O}_{S}\left(\sum 12 a_{i} D_{i}\right) \otimes J_{\infty}$.

$D_{i}$ are irreducible components of $\Sigma$ and $0 \leq a_{i}<1$ the rational numbers corresponding to the type of singularities over the general point of $D_{i}$ and are described explicitly in $[K d]$.

If we write $J_{\infty}=\sum b_{j} B_{j}$, then $\mathbf{J}$ has a pole of order $b_{j}$ along $B_{j}$.

For convenience, we give the following:

Definition. Let $\pi: X \rightarrow S$ be an elliptic fibration between smooth varieties. Assume that $\Sigma$ is a divisor with simple normal crossings. Define:

$$
\begin{aligned}
& \Delta_{X / S}=\sum 12 a_{i} D_{i}+(1 / 12) J_{\infty} \\
& \Lambda_{X / S}=\Delta_{X / S}+\sum \frac{m_{i}-1}{m_{i}} Y_{i}
\end{aligned}
$$

When there is no danger of confusion, we denote these simply by $\Delta$ and $\Lambda$ respectively. $\Lambda$ is thus a $\mathbb{Q}$-divisor.

Note that $[\Lambda]=0$, where $[x]$ denotes the integral part of $x$ (see 0.2$) ; 12 m \Lambda_{X / S}$ is a divisor supported on $\Sigma_{X / S}$.

In this notation we have

$$
m K_{X}=\pi^{*} m\left(K_{S}+\Lambda\right)+m E-m G
$$

Theorem 0.3 [G1]. Let $X_{0} \rightarrow S_{0}$ be an elliptic threefold which is not uniruled.

Then there exist a birationally equivalent fibration $\bar{\pi}: \bar{X} \rightarrow \bar{S}$, such that $\bar{X}$ has at worst terminal and $\bar{S} \log$ terminal singularities. Furthermore $K_{\bar{X}}$ is nef and $K_{\bar{X}} \equiv \bar{\pi}^{*}\left(K_{\bar{S}}+\bar{\Lambda}\right) ; \bar{\Lambda}$ is the $\mathbb{Q}$-divisor defined below.

Sketch of the proof. Let $\tilde{X} \rightarrow \tilde{S}$ be a birational equivalent fibration of smooth varieties. Let $X_{1} \rightarrow S$ be a flat model of the fibration and $X$ a non singular model of $X_{1}$. Denote by $\pi: X \rightarrow S$ the induced fibration. Assume that $\Sigma_{X / S}$ (the ramification divisor of $\pi$ ) is a divisor with simple normal crossings.

Since $X$ is not uniruled, $\kappa(X)$ is non-negative [Mi] and on some suitable birational model, some multiple of $K_{S}+\Lambda$ is an effective divisor. Then there exists a unique surface $\bar{S}$, a divisor $\bar{\Lambda}$ and a morphism $\phi: S \rightarrow \bar{S}$ such that $K_{\bar{S}}+\bar{\Lambda}$ is nef and $\phi^{*}\left(K_{\bar{S}}+\bar{\Lambda}\right)+\sum c_{i} \Gamma_{i}$ is the Zariski decomposition of $K_{S}+\Lambda$ [Za], [Sa]. Furthermore $\bar{\Lambda}=\phi_{*}(\Lambda)$, and $\bar{S}$ has at worst log terminal singularities: $\phi$ is a log terminal contraction, because $[\Lambda]=0$ [KMM, §5.1.5.].

In particular if $K_{S}+\Lambda$ is not nef, then there exists a log extremal curve $\Gamma_{1}$ such that $\left(K_{S}+\Lambda\right) \cdot \Gamma_{1}<0$, and a morphism $\phi_{1}: S \rightarrow S_{1}$. We successively construct the objects $\phi_{i}: S_{i-1} \rightarrow S_{i}$ where $\phi_{i}$ is the log extremal contraction associated to the log 
extremal curve $\Gamma_{i}$. All such $\Gamma_{i}$ are rational curves [Ka4], [Br]. $\phi$ is the composition of these log extremal contractions (a log contraction). Now we can apply relative Mori's theory [Mo] to the fibration $X \rightarrow \bar{S}$, and this concludes the proof.

Given $S$ and $\Lambda$ as in (0.3), $\bar{S}$ is uniquely determined [Sa, Thm 1.4]. However, the parameter surface of a minimal elliptic fibration is not uniquely determined by the birational equivalence class of the fibration:

Proposition 0.4. Let $\bar{\pi}_{i}: \bar{X}_{i} \rightarrow \bar{S}_{i}, i=1,2$ be 2 birationally equivalent elliptic fibrations with $\bar{X}_{i}$ minimal and $K_{\bar{X}_{i}} \equiv \bar{\pi}^{*}\left(K_{\bar{S}_{i}}+\bar{\Lambda}_{i}\right)$, $\forall i$. Denote by $\varphi: \bar{S}_{1} \rightarrow \bar{S}_{2}$ the corresponding birational map. Then $\left(K_{\bar{S}_{i}}+\bar{\Lambda}_{i}\right) \cdot \Gamma=0$, for every curve $\Gamma$ which is $\varphi$ or $(\varphi)^{-1}$-exceptional.

Proof. Let $q_{i}: Y \rightarrow \bar{X}_{i}$ the a resolution of the graph of the map $\mu: \bar{X}_{1} \rightarrow \bar{X}_{2}$. Then $q_{1}^{*}\left(K_{\bar{X}_{1}}\right)=q_{2}^{*}\left(K_{\bar{X}_{2}}\right)[\mathrm{Ko}, 4.4]$.

Consider a curve $\Gamma \varphi$-exceptional and any curve $C_{1} \subset \bar{X}_{1}$ dominating $\Gamma$. Let $\tilde{C}$ be the strict transform of $C_{1}$ on $Y$. Then by commutativity of the diagram:

$$
0=\left(K_{S_{2}}+\Lambda_{2}\right) \cdot\left(\bar{\pi}_{2} \cdot q_{2}\right)_{*}(\tilde{C})=K_{\bar{X}_{2}} \cdot\left(q_{2 *} \tilde{C}\right)=K_{\bar{X}_{1}} \cdot C_{1}=\left(K_{S_{1}}+\Lambda\right) \cdot \Gamma
$$

More generally the following holds:

Propostion 0.5. Let $\bar{\pi}_{2}: \bar{X}_{2} \rightarrow \bar{S}_{2}$ be an elliptic fibrations with $\bar{X}_{2}$ minimal. Let $\bar{\pi}: \bar{X}_{1} \rightarrow \bar{S}_{1}$ be a minimal model of $\bar{X}_{2}$ obtained as in (0.3). Although the birational map $\bar{X}_{2} \rightarrow S_{1}$ is not in general an elliptic fibration, there exists a birational morphism $\tau: \bar{S}_{1} \rightarrow \bar{S}_{3}$ such that the induced birational map $\rho: \bar{X}_{2} \rightarrow \bar{S}_{3}$ is not only a morphism but an elliptic fibration.

Furthermore, for every curve $\Gamma$ which is $\tau$ exceptional, there is a curve $C \subset \bar{X}_{1}$ dominating $\Gamma$ included on the exceptional locus of the birational map $\bar{X}_{1} \rightarrow \bar{X}_{2}$ and additionally $\left(K_{\bar{S}_{1}}+\bar{\Lambda}\right) \cdot \Gamma=0$.

Proof. Then statement is obvious if $\bar{S}_{2} \rightarrow \bar{S}_{1}$ is a morphism. Otherwise consider the graph:

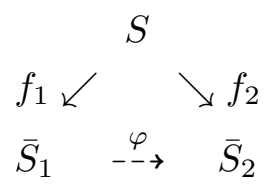

Let $F \subset S$ be the union of the exceptional locus of $f_{1}$ and $f_{2}$. Since the question is local we can assume $F$ connected. Let $V \subset \bar{S}_{3}$ be an open set contaning $f_{1}(F)$.

Consider the set of curves $\left\{C_{i}\right\} \subset \bar{X}_{i}$ which are included in the exceptional locus of $\mu$ and dominate a curve $\Gamma \subset f_{1}(F)$. Then $0=K_{\bar{X}_{i}} \cdot C_{i}=\left(K_{S_{1}}+\Lambda_{1}\right) \cdot \Gamma,[\mathrm{Ko}$, 
4.5]. Let $\tau: \bar{S}_{1} \rightarrow \bar{S}_{3}$ the contraction of all such curve $\Gamma$ and

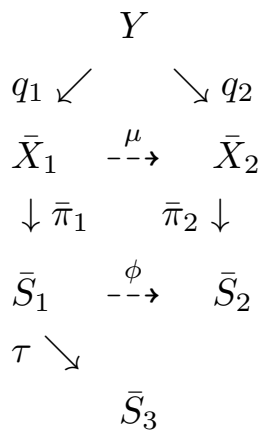

be a resolution of the graph of the map $\mu: \bar{X}_{1} \rightarrow-\bar{X}_{2}$. Then

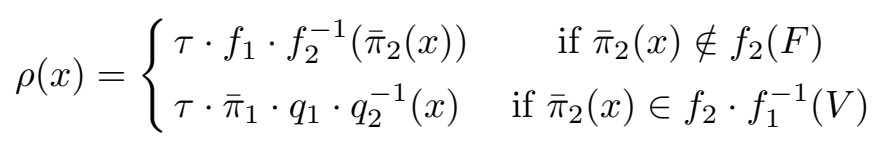

defines a rational elliptic fibration $\bar{X}_{2} \rightarrow \bar{S}_{3}$. We need to show that $\rho$ is actually a morphism. Assume that $P \in \bar{X}_{2}$ is a fundamental point of $\rho$ and let

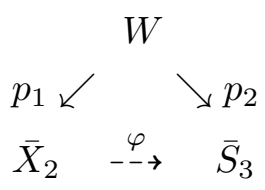

be the corresponding graph: note that $p_{1}$ is a birational map. The commutativity of the diargam and Zariski's Main Theorem imply that $p_{1}^{-1}(P)$ is connected and has dimension 0 , see for example $[\mathrm{Ha} 2,5.2]$. Then $\rho$ is actually a morphism.

Since two-dimensional log terminal singularities are quotient [Ka4], the exceptional graphs on the minimal resolution of $\bar{S}$ must be part of the Brieskorn's list of surface quotient singularities $[\mathrm{Br}]$.

If the fibration does not have multiple fibres singularites of $\bar{S}$ occur when the $\mathbb{Q}$-divisor $\Delta=\pi_{*}\left(K_{X / S}\right)$ is not nef.

Because of the connection with the $C_{n, m}$ conjecture [Ka6] and the birational classification of varieties, different notions of "positivity" of the direct image of the relative dualizing sheaf have been introduced and studied. Here is a list of some "positivity" results that are relevant to the case of elliptic fibrations:

Theorem [Ka1]. Let $g: T \rightarrow W$ be any surjective morphism between non singular projective varieties where the ramification divisor $\Sigma$ has simple normal crossings and the local monodromies of $\mathbb{R} g_{*} \mathbb{C}_{X_{0}}$ are unipotent. Then $g_{*}\left(K_{T / W}\right)$ is locally free and semipositive. 
Theorem $[\mathbf{K z}]$. An algebraic fiber space with simple normal crossings branching has unipotent monodromies if it is semistable in codimension 1.

These result show that all monodromies being unipotent, or "most" fibers reduced with simple normal crossings, is a sufficient condition for $\pi_{*} K_{X / S}$ to be nef; in the case of an elliptic fibration of a surface over a curve, $\pi_{*} K_{X / S}=\Delta$ has always non-negative degree.

Note that, in the case of invertible sheaves, semipositive is equivalent to nef (see, for example [Ha1] and/or [Ka3]).

\section{Notations.}

Throughout this paper, $\pi: X \rightarrow S$ denotes an elliptic fibration between smooth varieties, $\operatorname{dim}(X)=3$ and $\Sigma=\Sigma_{X / S}$ a divisor with simple normal crossings.

(0.4) We assume $S$ to be simple normal crossings minimal, that is, any contraction $\phi: S \rightarrow S_{1}$ of a log extremal curve leads to $S_{1}$ singular or $\Sigma_{X / S_{1}}$ not simple normal crossings.

(0.5) Let $E$ be an irreducible component of $\Sigma$. By abuse of notation we say that $E$ is of type $*$ if $\pi^{-1}(P)$ is a fiber of Kodaira type $*$, for general $P \in E$.

(0.8) A string of lenght $r$ is a union $C=\cup C_{i}$ of smooth rational curves $C_{i}$, such that $C_{i} \cdot C_{j}=1$, for $|i-j|=1$ and $C_{i} \cdot C_{j}=0$, for $|i-j|>1$. A Hirzebruch-Jung string is a string in the above sense with $C_{i}^{2} \leq-2, \forall i$.

(0.9) Consider a string of log extremal curves of length $r$. Set $S=S_{0}$ and denote by $\psi_{i}: S_{i-1} \rightarrow S_{i}, 1 \leq i \leq r$ the contraction of the log extremal curve $E_{i} \subset S_{i-1}$, and by $\psi: S \rightarrow S_{r}$ the composition of the contraction morphisms. Set $E_{k, 0}=E_{k}$ and let $E_{k, h} \subset S_{k-h-1}$ be the strict transform of $E_{k, h-1}$.

The string is of type $\langle n, q\rangle$, if $\langle n, q>$ represents the continued fraction:

$$
\frac{n}{q}=b_{1}-\frac{1}{b_{2}-\frac{1}{\cdots-\frac{1}{b_{r}}}}
$$

and $\left(E_{1}\right)^{2}=-b_{1},\left(E_{2,1}\right)^{2}=-b_{2}, \cdots,\left(E_{r, r-1}\right)^{2}=-b_{r}$.

For short we will write :

$$
\frac{f_{k}}{g_{k}}=b_{k}-\frac{1}{b_{k-1}-\frac{1}{\cdots-\frac{1}{b_{1}}}}
$$

where $f_{k}$ and $g_{k}$ are positve integers defined by induction as follows:

$$
\left\{\begin{array} { r } 
{ f _ { 1 } = b _ { 1 } } \\
{ g _ { 1 } = 1 }
\end{array} \quad \text { and } \quad \left\{\begin{array}{r}
b_{k} f_{k-1}-g_{k-1}=f_{k} \\
f_{k-1}=g_{k} .
\end{array}\right.\right.
$$

In fact, $\frac{f_{k}}{g_{k}}=\frac{b_{k} f_{k-1}-g_{k-1}}{f_{k-1}}=b_{k}-\frac{g_{k-1}}{f_{k-1}}$.

Note that $\frac{g_{r}}{f_{r}}=\frac{q^{\prime}}{n}$, where $q^{\prime}<n$ and $q q^{\prime} \equiv 1 \bmod n$. 
(0.11) $K_{k}$ will denote $K_{S_{k}}, \Delta=\Delta_{0}$, and $\Delta_{k}$ will stand for $\left(\psi_{k-1}\right)_{*}\left(\Delta_{S_{k-1}}\right)$. Write

$$
\Delta_{k-1}=\psi_{k}^{*}\left(\Delta_{k}\right)+\alpha_{k} E_{k}, \quad K_{k-1}=\psi_{k}^{*}\left(K_{k}\right)+\beta_{k} E_{k},
$$

and for some rational numbers $\alpha_{k}$ and $\beta_{k}$.

Thus:

$$
\begin{aligned}
& K_{k-1} \cdot E_{k}=\left[\psi_{k}^{*}\left(K_{k}\right)+\beta_{k} E_{k}\right] \cdot E_{k}=\beta_{k} E_{k}^{2} \\
& \Delta_{k-1} \cdot E_{k}=\left[\psi_{k}^{*}\left(\Delta_{k}\right)+\alpha_{k} E_{k}\right] \cdot E_{k}=\alpha_{k} E_{k}^{2}
\end{aligned}
$$

(0.12) If $S \rightarrow \bar{S}$ is a $\log$ contraction, with $\bar{S}$ singular and $S$ smooth, we say that $S$ is the log extremal resolution of $\bar{S}$. Throughall this paper all the singular surfaces $\bar{S}$ are obtained by contracting log extremal curves and thus there exists a log extremal resolution.

(0.13) If the rational map $\mathbf{J}: S \rightarrow \mathbb{P}^{1}$ induced by the elliptic fibration is constant along a divisor $E \in \Sigma$, with value $\lambda$, we say that $E$ has $J$-invariant $\lambda$, or, $J(E)=\lambda$.

\section{Properties.}

The following is a list of first properties of log extremal curves (see the proof of Theorem 0.3):

\section{Proposition 0.14 .}

(1) If $\Lambda \cdot E \geq 0$, then $\left(K_{S}+\Lambda\right) \cdot E<0$ if and only if $K_{S} \cdot E<0$, that is $E$ contracts to a smooth point.

(2) Let $\bar{E}$ be a log extremal curve on a singular surface $\bar{S}$. If $\bar{\Lambda} \cdot \bar{E} \geq 0$, then the contraction of $\bar{E}$ "improves the singularity".

(3) $\Delta \cdot E \in \mathbb{Z}$, for every curve $E$ on $S$, because $\Delta$ is the divisor associated to the line bundle $\pi_{*}\left(K_{X / S}\right)$.

(4) If $\psi: S \rightarrow S_{1}$ is a log-contraction to a smooth point, then $\left(K_{S_{1}}+\Delta_{1}\right)$ is a Cartier divisor; consequently $\Delta_{1} \cdot E$ is an integer for any curve $E$ on $\bar{S}$.

Proof. (1),(3), (4) follow from 0.3.

(2) Let $\phi: S \rightarrow \bar{S}$ be the minimal log extremal resolution (see 0.12). If $E$ denotes the strict transform of $\bar{E}$, then the assumptions imply $K_{S} \cdot E<K_{\bar{S}} \cdot \bar{E}<0$.

Thus $\phi$ can can be re-factored, first contracting $E$ to a smooth point.

\section{Strategy}

In $\S 2$ and $\S 4$ we investigate the singularities arising from contractions of log extremal curves in $\Sigma-\Sigma^{m}$.

From now on, $\phi: S \rightarrow \bar{S}$ denotes the contraction of all the log exceptional curves $\Gamma$ in $\Sigma-\Sigma^{m} ;$ then $\left(K_{S}+\Lambda\right) \cdot \Gamma=\left(K_{S}+\Delta\right) \cdot \Gamma$. The key point in the analysis of such contractions is that $\Lambda \cdot \Gamma \geq \Delta \cdot \Gamma$ and that $\Delta \cdot \Gamma$ has to be an integer $[0.14$, part $3]$. 
Our strategy is to factor the contraction morphism $\phi: S \rightarrow \bar{S}$ into 3 morphisms $\phi=\varphi_{1} \cdot \varphi_{2} \cdot \varphi_{3}$ :

$$
\varphi_{1}: S \rightarrow S_{1}, \varphi_{2}: S_{1} \rightarrow T, \varphi_{3}: T \rightarrow \bar{S}
$$

(1) $\varphi_{1}$ denotes the composition of contractions of successively log extremal curves to smooth points and $S_{1}$ is a smooth surface. Set $\Delta_{1}=\varphi_{1 *}(\Delta)$.

(2) $\varphi_{2}$ is the morphism associated to the contraction of log extremal HirzebruchJung curves. $T$ is a singular surface and $S_{1}$ is its minimal resolution. Set $\Delta_{T}=\varphi_{2 *}\left(\Delta_{1}\right)$.

(3) $\varphi_{3}$ denotes the contraction of all the remaining log extremal curves. Then $\bar{\Delta}=\left(\varphi_{3}\right) *\left(\Delta_{T}\right)=\phi_{*}(\Delta)$.

The order of contraction of log extremal curves is arbitrary: this one has been chosen because of its logical convenience. The argument is nevertheless general, because the minimal surface (with log terminal singularities) associated to $S$ and $\Lambda$ [Sa, Thm 1.4].

First we will set up a combinatoric contraction algorithm for (1) and (2) (in 2.2., 2.5.) and then prove that all the curves contracted by $\varphi_{3}$ have self-intersection $(-1)$ on the minimal resolution $S_{1}$. We will show that if $E_{i}$ is contracted by $\varphi_{3}$, then $\left(K_{T}+\Delta_{T}\right) \cdot \hat{E}_{i}=0$, where $\hat{E}_{i}$ is the strict transform of $E_{i}$ on $S_{1}$. These two properties will allow us to re-factor $\varphi_{2} \cdot \varphi_{3}$, first contracting $\hat{E}_{i}$ to a smooth surface $S_{i_{1}}^{\prime}$ and then applying the previous algorithm to the remaining contractions. This process thus produces an iterative contraction algorithm; eventually $\phi$ is factored as:

$$
\varphi_{1}^{\prime}: S \rightarrow S^{\prime} \text { and } \varphi_{2}^{\prime}: S^{\prime} \rightarrow S_{2}^{\prime}=\bar{S}
$$

where $S^{\prime}$ is the smooth minimal resolution of $\bar{S}, \Sigma_{X / S^{\prime}}$ is not a divisor with simple normal crossings (if $S \neq S^{\prime}$ ) and $\varphi_{2}^{\prime}$ contracts only Hirzebruch-Jung strings.

Note that $\varphi_{1}^{\prime}$ is not a $\log$ extremal contraction.

The contraction of $\varphi_{1}$ (respectively $\varphi_{2}, \varphi_{3}$ ) will be denoted by the first (respectively second and third) step.

In $\S 2$ and $\S 4$ we state and prove the major points in the algorithm; the technical details are treated in $\S 5$.

\section{The first two steps}

First we consider the case of a log extremal curve on a smooth surface with $J$ invariant $\infty$.

The following proposition says that $\log$ extremal strings with $J=\infty$ do not contract to singular points of $\bar{S}$.

Proposition 2.2., 2.1 and 2.5. together form the basic for the combinatorics of our contraction algorithm. In fact, 2.5. states that a log extremal curve $E_{1}$ on a smooth surface must have intersection -1 with $\left(K_{S}+\Delta\right)$ while 2.5. gives bounds 
on the self-intersection number of $E_{1}$ and on the sum of the coefficients (in $\Delta$ ) of the intersecting curves.

The second parts of 2.2. and 2.5. tell us how to continue the contraction algorithm in (1) and (2).

Proposition 2.1. Let $\pi: X \rightarrow S$ be an elliptic fibration such that the $J$ invariant function extend to a morphism $\mathbf{J}: S \rightarrow \mathbb{P}^{1}$. Assume that $\mathbf{J}$ has a pole over a log extremal curve $B_{1}$ and denote by $\psi_{1}: S \rightarrow S_{1}$ the corresponding contraction.

(a) $\mathbf{J}: S_{1} \rightarrow \mathbb{P}^{1}$ is a morphism $\psi_{1}^{*}\left(J_{\infty}^{1}\right)=J_{\infty}$, where $J_{\infty}^{1}$ denotes the divisor of poles of $\mathbf{J}$ on $S_{1}$.

(b) If $S$ is smooth and $\Delta \cdot C \in \mathbb{Z}$, for all 1-cycle $C$ on $S$, then $\left(B_{1}\right)^{2} \geq-2$. $\left(B_{1}\right)^{2}=-2$ only if the fiber over the general point of $B_{1}$ is of type $I_{a}^{*}$. If $\left(B_{1}\right)^{2}=-1$, then $\Delta_{1} \cdot C \in \mathbb{Z}$, for all 1-cycle $C$ on $S_{1}$.

(c) Let $B_{1}$ and $S_{1}$ be as in (b), with $\left(B_{1}\right)^{2}=-2$. Let $B_{2,1} \in \Sigma$ be another curve such that $B_{2,1} \cdot B_{1}=1$ and $B_{2}=\left(\psi_{1}\right)_{*}\left(B_{2,1}\right)$ is a log extremal curve on $S_{1}$. Denote by $\psi_{2}$ : $S_{1} \rightarrow S_{2}$ the corresponding contraction. Then $S_{2}$ is smooth and $\left(K_{S}+\Delta\right) \cdot B_{2,1}=0$.

Recall that is $S$ is the smooth surface image of log extremal contractions, then $\Delta \cdot C \in \mathbb{Z}$, for all 1-cycle $C$ on $S$. Note also that this agrees with $[\mathrm{Kz}]$.

Proof.

(a) Consider $H$ a smooth irreducible element of $J_{\infty}$. Since $H$ is a fiber of $\mathbf{J}$, $H \cdot B_{1}=0$. Thus the statement follows directly from the definition.

(b) Write

$$
\Delta=\sum a_{i} D_{i}+\delta\left(B_{1}\right) B_{1}+(1 / 12) J_{\infty},
$$

where $D_{i} \neq B_{1}, \forall i ; \delta\left(B_{1}\right)=1 / 2$ when $B_{1}$ is of type $I_{a}^{*}$ or $\delta\left(B_{1}\right)=0$ when $B_{1}$ is of type $I_{a}[\mathrm{Kd}]$.

Set $\Delta_{1}=\psi_{*}(\Delta)$ and $\bar{D}_{i}=\psi_{*}\left(D_{i}\right)$. Thus $\Delta_{1}=\sum a_{i} \bar{D}_{i}+(1 / 12) J_{\infty}$. We will prove the statement comparing $\Delta$ and $\psi_{1}^{*}\left(\Delta_{1}\right)$. Set:

$$
\begin{aligned}
\Delta & =\psi_{1}^{*}\left(\Delta_{1}\right)+\alpha_{1} B_{1}, \quad \alpha_{1} \geq 0 \\
D_{i} & =\psi_{1}^{*}\left(\bar{D}_{i}\right)-\gamma_{i} B_{1}, \gamma_{i} \geq 0
\end{aligned}
$$

It follows that $(1 / 2) \delta=\sum a_{i} \gamma_{i}+\alpha_{1}$ and $0 \leq \alpha_{1} \leq 1 / 2$, with $\alpha_{1}=1 / 2$ only when $\delta=1 / 2$ and $\gamma_{i}=0, \forall i$. Then (5.5) applies and $1 / 2 \geq \alpha=1+\frac{1}{\left(B_{1}\right)^{2}}$. This shows that $\left(B_{1}\right)^{2} \geq-2$ and $\left(B_{1}\right)^{2}=-2$ only when $B_{1}$ is of type $I_{a}^{*}$ and $\gamma_{1}=0, \forall i$.

(c) Set $B_{2,1}^{2}=-b_{2}$. Then

$$
\Delta_{1} \cdot B_{2}=\Delta \cdot B_{2,1}-1 / 2 \geq 1-1 / 2=1 / 2 .
$$

Since $K_{S}=\psi^{*}\left(K_{S_{1}}\right)$ and $B_{2}$ is log extremal, the above inequality implies

$$
0>\left(K_{S_{1}}+\Delta_{1}\right) \cdot B_{2}=b_{2}-2+\Delta_{1} \cdot B_{2} \geq b_{2}-3 / 2 .
$$

Thus $b_{2}=1$; furthermore $\Delta_{1} \cdot B_{2,1}=1$ and $\left(K_{S}+\Delta\right) \cdot B_{2,1}=0$. 
Proposition 2.2. Let $\left\{E_{1}, E_{2,1}, \ldots, E_{r, r-1}\right\}$ be a string of log extremal curves on a smooth surface $S$. ( $\Sigma$ is not necessarily simple normal crossing.) Assume that $\Delta \cdot E_{j, j-1}$ is an integer, $\forall j$ and that the string is represented by $<n, q>$. Then in the notation of (0.9) we have:

(1) $\left(K_{S}+\Delta\right) \cdot E_{1}=-1$ and $\left(K_{S}+\Delta\right) \cdot E_{j, j-1}=0, \forall j, 2 \leq j \leq r$.

(2) $\left(K_{S}+\Delta\right) \cdot \Delta=\left(K_{S_{r}}+\Delta_{r}\right) \cdot \Delta_{r}+\frac{1}{n}-1$.

Proof of Proposition 2.2..

(1) By (5.1), (5.2), (5.5) and (0.4) we have

$$
\left(K_{S}+\Delta\right) \cdot E_{1}=\left(\alpha_{1}+\beta_{1}\right)\left(E_{1}\right)^{2}
$$

and thus $\frac{1}{f_{1}} \frac{-f_{1}}{g_{1}}=-1$.

Similarly, for any $k \geq 2$ we can write:

$$
\left(K_{S}+\Delta\right) \cdot E_{k, k-1}=\left(\alpha_{k}+\beta_{k}\right)\left(E_{k}\right)^{2}+\alpha_{k-1}+\beta_{k-1}=\frac{-f_{k}}{f_{k} g_{k}}+\frac{1}{f_{k-1}}=0 .
$$

(2) By $(0.11),\left(K_{S}+\Delta\right) \cdot \Delta=\left(K_{S_{r}}+\Delta_{r}\right) \cdot \Delta_{r}+\sum_{k} \alpha_{k}\left(\alpha_{k}+\beta_{k}\right)\left(E_{k}\right)^{2}$.

Substituting the expressions for $\alpha_{k}$ and $\beta_{k}$ derived in 5.3. and 5.5. we have

$$
\sum_{k=1}^{r} \alpha_{k}\left(\alpha_{k}+\beta_{k}\right)\left(E_{k}\right)^{2}=\sum_{k=1}^{r} \frac{f_{k}-g_{k}}{f_{k}} \cdot \frac{1}{f_{k}} \cdot \frac{-f_{k}}{g_{k}}=\sum_{k=1}^{r}-1 / g_{k}+1 / f_{k} .
$$

By $(0.11) \sum_{k}\left(-1 / g_{k}+1 / f_{k}\right)=-(1-1 / n)$ and thus

$$
\left(K_{S}+\Delta\right) \cdot \Delta=\left(K_{S_{r}}+\Delta_{r}\right) \cdot \Delta_{r}-(1-1 / n) .
$$

The following corollary is a particular case of Theorem 2.5.: however, it is also an immediate application of 2.2 .

Corollary 2.3. Let $\left\{E_{1}, \ldots, E_{r, r-1}\right\}$ be a Hirzebruch-Jung string of log extremal curves on a smooth surface $S$ represented by $\langle n, q\rangle$. Assume that the string is a connected component of $\Sigma$. Then:

(1) $\Delta=\left(1-\frac{g_{r}}{f_{r}}\right) E_{r, r-1}+\ldots\left(1-\frac{g_{2}}{f_{r}}\right) E_{2,2-1}+\left(1-\frac{g_{1}}{f_{r}}\right) E_{1}$.

(2) $r \leq 5 ;<n, q>$ and $\left(a_{1}, \ldots, a_{r}\right)$ (the coefficients of $\left\{E_{1}, \ldots E_{r, r-1}\right\}$ in $\Delta$ ) are described in the table below.

$\left|\begin{array}{ccccccc}n & q & a_{1} & a_{2} & a_{3} & a_{4} & a_{5} \\ 2 & 1 & 1 / 2 & & & & \\ 3 & 1 & 2 / 3 & & & & \\ 6 & 1 & 5 / 6 & & & & \\ 4 & 1 & 3 / 4 & & & & \\ 4 & 3 & 3 / 4 & 1 / 2 & 1 / 4 & & \\ 3 & 2 & 2 / 3 & 1 / 3 & & & \\ 6 & 5 & 5 / 6 & 2 / 3 & 1 / 2 & 1 / 3 & 1 / 6\end{array}\right|$


Proof. Part (1) follows from 2.2 and 5.5. It follows also that $a_{r}=\alpha_{r}=1-$ $\frac{g_{r}}{f_{r}}=1-\frac{q^{\prime}}{n}$, where $q^{\prime}<n$ and $q q^{\prime} \equiv 1 \bmod n$. Note that $\left\{E_{1}, \ldots, E_{r, r-1}\right\} \subset$ $\Sigma$ and that $p$ singular implies $\mathbf{J}\left(E_{j}\right) \neq \infty$, for every $j$ (see 2.1). Hence $a_{r} \in$ $\{1 / 2,2 / 3,5 / 6,3 / 4,1 / 4,1 / 3,1 / 6\}[\mathrm{Kd}]$.

2.3 describes the log extremal Hirzebruch-Jung strings which are connected components of $\Sigma_{X / S}$. The last part of this section contains numerical conditions which, together with 2.1 and 2.2., describe the contraction of any log extremal string.

\section{Proposition 2.4.}

(1) Let $E_{1}$ be a log extremal curve of self-intersections $-b_{1}$.

Write $\Delta=e_{1} E_{1}+\sum c_{i} C_{i}$, where each $C_{i}$ is an irreducible curve, $c_{i}$ and $e_{1}$ are the rational number described in 0.2 .

$$
\begin{aligned}
& \text { If }\left(K_{S}+\Delta\right) \cdot E_{1}=-1, \text { then } \\
& \qquad 0 \leq \sum_{i} c_{i}\left(C_{i} \cdot E_{1}\right)=1-\left(1-e_{1}\right) b_{1}<e_{1}
\end{aligned}
$$

In particular, $b_{1} \leq\left(1-e_{1}\right)^{-1}$.

(2) Let $E_{2,1}$ be another $\log$ extremal curve such that $E_{1} \cdot E_{2,1}=1$ and $\left(E_{2,1}\right)^{2}=$ $-b_{2}$ (the notation is as in 0.9).

Write $\Delta=e_{1} E_{1}+e_{2} E_{2}+\sum c_{i} C_{i}$. If $\left(K_{S}+\Delta\right) \cdot E_{2,1}=0$, then

$$
0 \leq \sum_{i} c_{i}\left(C_{i} \cdot E_{2,1}\right)=2-\left(1-e_{2}\right) b_{2}-e_{1}
$$

In particular, $b_{2} \leq \frac{2-e_{1}}{1-e_{2}}$.

Proof. $E_{1}$ and $E_{2,1}$ are rational curves, hence: $\Delta \cdot E_{1}=\left(K_{S}+\Delta\right) \cdot E_{1}-b_{1}+2$; $\Delta \cdot E_{2,1}=\left(K_{S}+\Delta\right) \cdot E_{2,1}-b_{2}+2$.

(1) $\left(K_{S}+\Delta\right) \cdot E_{1}=-1 \Longleftrightarrow \Delta \cdot E_{1}=1-b_{1}$ thus

$$
\begin{aligned}
1-b_{1} & =-e_{1} b_{1}+\sum_{i} c_{i}\left(C_{i} \cdot E_{1}\right) \quad \text { i.e. } \\
1-\left(1-e_{1}\right) b_{1} & =\sum_{i} c_{i}\left(C_{i} \cdot E_{1}\right) \geq 0 .
\end{aligned}
$$

In particular $b_{1} \leq\left(1-e_{1}\right)^{-1}$.

(2) Similarly,

$$
\begin{array}{r}
e_{1}-e_{2} b_{2}+\sum_{i} c_{i}\left(C_{i} \cdot E_{2,1}\right)=\Delta \cdot E_{2}=2-b_{2} \quad \text { i.e } \\
2-\left(1-e_{2}\right) b_{2}-e_{1}=\sum_{i} c_{i}\left(C_{i} \cdot E_{2,1}\right) \geq 0 .
\end{array}
$$

In particular $b_{2} \leq \frac{2-e_{1}}{1-e_{2}}$. 


\section{Proposition 2.5.}

Let $\left\{E_{1}, E_{2,1}\right\}$ be two log extremal curves on the smooth surface $S$. As in (0.9) we denote by $\psi_{1}: S \rightarrow S_{1}$ the contraction of $E_{1}$ and by $\psi_{2}: S_{1} \rightarrow S_{2}$ the contraction of $E_{2}$. If $S_{1}$ is smooth so is $S_{2}$.

Proof. By 2.1 we can assume $J\left(E_{1}\right) \neq \infty$.

Denote by $e_{1}\left(\right.$ resp $\left.e_{2}\right)$ the coefficient of $E_{1}\left(\right.$ resp $\left.E_{1,2}\right)$ in $\Delta$ and use the same notation of 2.4. Since $E_{1}$ contracts to a smooth point $-\left(b_{2}-1\right)=\left(E_{2}\right)^{2}$.

Assume that $b_{2}-1 \geq 2$ and derive a contradiction. By $2.2\left(K_{S_{1}}+\Delta_{1}\right) \cdot E_{2}=-1$ and

$$
\Delta_{1} \cdot E_{2}=-1-K_{S_{1}} \cdot E_{2}=-1+2-\left(b_{2}-1\right)=1-\left(b_{2}-1\right) .
$$

On the other hand, since $E_{1}$ contracts to a smooth point $\Delta \cdot E_{1}=0$ and $\Delta_{1} \cdot E_{2} \geq$ $e_{1}-e_{2}-\left(b_{2}-1\right) e_{2}$. Note also that $e_{1}-e_{2} \geq 0$. Combining the two expressions for $\Delta_{1} \cdot E_{1}$ we have:

$$
1-\left(b_{2}-1\right) \geq e_{1}-e_{2}-\left(b_{2}-1\right) e_{2} \text {, that is }
$$

$e_{1}-e_{2} \leq 1-\left(b_{2}-1\right)\left(1-e_{2}\right)$. If $b_{2}-1 \geq 2$, then $e_{1}-e_{2} \leq 2 e_{2}-1$ and thus $e_{1} \leq 3 e_{2}-1$. Recall that $S$ is assumed to be simple normal crossing minimal (0.4), and thus $e_{1}-e_{3} \geq 1 / 3$. Combining the two inequalities we get $e_{1} \leq 3 e_{1}-2$ or $1 \leq e_{1}$, which is a contradiction.

Proposition 2.5, which depends on 2.1, also implies 2.1 as a particular case.

Now we are ready to apply the previous computation in order to describe the log extremal curves contracted by $\varphi_{1}$ and $\varphi_{2}$.

Recall that:

(1) First Step $\varphi_{1}: S \rightarrow S_{1}$ is the contraction of all log extremal curves to smooth points.

(2) Second Step $\varphi_{2}: S_{1} \rightarrow T$ contracts all the chains of disjoint Hirzebruch-Jung curves.

(The case when $J$ has poles on the whole string is considered in (2.1); thus we can assume that the first curve to contract has $J$ invariant $\neq \infty$; furthermore, the first curve to contract is disjoint from the image of the previous exceptional smooth strings by Prop. 2.5.) $S_{1}$ is the minimal resolution of $T$.

\section{Theorem 2.6.}

After the first step (1) the possible singularities of $\Sigma_{X / T}$ coming from contractions of curves with $J \neq \infty$ are the one listed in Table 1 .

After the second step (2) the Hirzebruch-Jung strings which can occur are of type $<n, q>, n \leq 6$.

Furthermore the type of the corresponding singular fiber are listed in Table 2, 3, 5 .

Proof. If $J\left(E_{1}\right)=\infty$ then the statement follows form (2.1). Assume $J\left(E_{1}\right) \neq \infty$. The application of the contraction algorithm is straightforward and here we work out only the particular case of $e_{1}=2 / 3$. Further details are avaliable upon request. 
Recall that we assume $S$ to be simple normal crossing minimal. The notation is as in 2.4

Case 1: If $b_{1}=1$, then $\left(K_{S}+\Delta\right) \cdot E_{1}=-1$ and $\sum c_{i}=2 / 3(2.4)$. Thus $\left\{c_{1}, c_{2}, c_{3}\right\}=$ $\left\{\frac{1}{3}, \frac{1}{6}, \frac{1}{6}\right\}$. Again by 2.4 only $C_{1}$ can be $\log$ extremal. Set $C_{1}=E_{2,1}$. Then $0=$ $E_{2,1} \cdot \Delta_{1}=\frac{1}{3}+\frac{1}{6}+\frac{1}{6}$.

Case 2: $2 \leq b_{1}$. By 2.4., $b_{1} \leq 3$.

Case $2_{A} b_{1}=3$. The curve $E_{1}$ (type $I V^{*}$ ) has to be a connected component of $\Sigma_{X / S_{1}}$.

Case $2_{B} \quad b_{1}=2, \sum c_{i}=1 / 3$. Then $c_{1}=1 / 3($ type $I V)$, or $\left\{c_{1}, c_{2}\right\}=\left\{\frac{1}{6}, \frac{1}{6}\right\}$ (types $I I, I I) . C_{1}$ can be part of a log extremal Hirzebruch-Jung string only if it is of type $I V\left(2.4\right.$, part 2 and 2.2). Set $C_{1}=E_{2,1}$. Then $b_{2}=2$ and $\left\{E_{1}, E_{2,1}\right\}$ is a connected component of $\Sigma_{X / S_{1}}$.

In the following pages $r$ denotes the length of the string. The roman numbers denote the type of the singular fiber in the Kodaira classification, while the arabic ones, between parenthesis, are the self-intersection numbers of the exceptional curves.

The solid (resp. empty) circles represent the curves in $\Sigma_{X / T}$ which are (resp. are not) log extremal.

Note that except the $\langle 5,2\rangle$ and $\langle 5,3\rangle$ cases (where the exceptional curves have selfintersection $(-2,-3)$ and $(-3,-2))$, all the strings of length $\geq 2$ are of $(-2)$ curves. 


\section{Examples}

\section{Example 3.1 - 3.7.}

In the 2 following examples, $\zeta=e^{2 \pi i / n}$ is a root of unity and $E=\mathbb{C} /<1, \zeta>$ a smooth elliptic curve. Consider $\bar{X}=\left(\mathbb{C}^{2} \times E\right) / G$, where $G=\mathbb{Z} / n \mathbb{Z}$ and the action is specified below. In (1) and (2) $\bar{S}=\mathbb{C}^{2} / G$ has a singular point at the origin and $\bar{\pi}: \bar{X} \rightarrow \bar{S}$ is an elliptic threefold with terminal singularities. Let $\phi: S \rightarrow \bar{S}$ be the minimal resolution of $\bar{S}$ and $\pi: X \rightarrow S$ the resolution of the induced fibration:

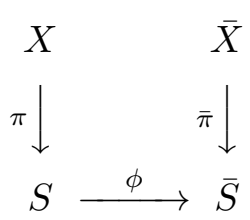

(1) Let $n=2,3,4,6$ and $G=\mathbb{Z} / n \mathbb{Z}$ act on $\left(z_{1}, z_{2}, e\right) \in\left(\mathbb{C}^{2} \times E\right)$ as follows: $\left(z_{1}, z_{2}, e\right) \mapsto\left(\zeta z_{1}, \zeta z_{2}, \zeta^{-1} e\right)$.

The exceptional graphs of $\phi$ are of type $<n, 1>$ and $\pi^{-1}(P)$ is a singular fiber with monodromy eingenvalue $e^{2 \pi i(n-i) / n}$ (corresponding respectively to the Kodaira types $\left.I_{0}^{*}, I V^{*}, I I I^{*}, I I^{*}\right)$.

(2) Let $n=3,4,6$ and $G=\mathbb{Z} / n \mathbb{Z}$ act on $\left(z_{1}, z_{2}, e\right) \in\left(\mathbb{C}^{2} \times E\right)$ as: $\left(z_{1}, z_{2}, e\right) \mapsto$ $\left(\zeta z_{1}, \zeta^{-1} z_{2}, \zeta e\right)$

The exceptional graphs of $\phi$ are of type $<n, n-1>$ and the corresponding divisors of singular fibers are: $I V^{*}-I V(n=3) ; I I I^{*}-I_{0}^{*}-I I I(n=4)$ and $I I^{*}-I V^{*}-I_{0}^{*}-I V-I I(n=6)$.

Example 3.8: $q=1, n=5$.

Let $\Gamma$ be the rational normal curve in $\mathbb{P}^{5}$ given by the embedding $[x, y] \mapsto$ $\left[y^{5}, y^{4} x, y^{3} x^{2}, y^{2} x^{3}, y x^{4}, x^{5}\right]$. Let $T$ be the projective cone over $\Gamma$ and $\tilde{T}$ its minimal resolution with exceptional divisor $D$.

The automorphism $g:[x, y] \mapsto[x, \zeta y]$ of $\mathbb{P}^{1}$ (where $\zeta=e^{2 \pi i / 6}$ ) induces the automorphism of the cone:

$$
\left(z_{1}, z_{2}, z_{3}, z_{4}, z_{5}, z_{6}\right) \mapsto\left(\zeta^{5} z_{1}, \zeta^{4} z_{2}, \zeta^{3} z_{3}, \zeta^{2} z_{4}, \zeta z_{5}, z_{6}\right)
$$

It easy to see that $L=\left\{z_{j}=0\right\}, j=1,2,3,4,5$ is a line entirely fixed by $G$, the group generated by $g ; D$ is invariant under $G$ and the unique fixed point $P \in D$ generates a singularity on $\tilde{T} / G$.

Let $\rho: \tilde{S} \rightarrow \tilde{T}$ be the blow up at $P$, with exceptional divisor $\tilde{F}\left(\tilde{F}^{2}=-6\right) ; \tilde{D}$ the strict trasform of $D$ has self-intersection -6 . $\tilde{S} / G$ is then a smooth surface and $\tilde{D} / G$ can be contracted to a smooth point $\chi: \tilde{S} \rightarrow S$; furthermore $F^{2}=\chi_{*}(\tilde{F})^{2}=-5$.

We can extend the action of $g$ to $T \times E$ by sending $e \mapsto \zeta e$. The ramification divisor of the induced fibration $\pi: X \rightarrow S$ is supported on $F$ and $L$. A computation in local coordinates shows that the singular fiber are respectively of type $I I^{*}$ and $I I$ and that $X$ has terminal singularities. 


\section{More contractions}

Now, the particular goal is to investigate the case of new contractions, and in particular look for new exceptional graphs. From now on, $E$ is a log extremal curve on $T$ and $\hat{E}$ the strict transform of $E$ on $S_{1}$.

The curves that can contract now (and could not have been contracted with the first step $\varphi_{1}: S \rightarrow S_{1}$ and the second step $\varphi_{2}: S_{1} \rightarrow T$ ) belong to the following classes:

(i) curves $E \notin \Sigma_{X / T}$ whose image pass through the singularities of $T$,

(ii) curves intersecting 1 or more exceptional graphs, but not part of a HirzebruchJung string. [These curves are represented by empty cirles in the previous pages.]

The following is an example of case (i).

Example. Let $\pi_{2}: X_{2} \rightarrow S_{2}$ be an elliptic fibration between smooth varieties $(\operatorname{dim}(X)=3)$; let $C \in \Sigma_{X_{2} / S_{2}}$ such that $\pi_{2}^{-1}(p)$ is a singular fiber of type $I_{0}^{*}$, for general $p \in C$. [In a neighborood of $p$ the local equation for $X_{2}$ is given by the desingularization of the Weierstrass equation: $y^{2}=x^{3}+s^{2} t^{2} x+s^{2} t^{2}$.]

Let $\psi_{2}: S_{1} \rightarrow S_{2}$ be the blowup of $S_{2}$ at $p$ with exceptional divisor $E_{2}$. Denote by $\pi_{1}: X_{1} \rightarrow S_{1}$ the resolution of the induced fibration. The singular fiber over the general point of $E_{2}$ is again of type $I_{0}^{*}$.

Let $\psi_{1}: S_{0} \rightarrow S_{1}$ be the blowup of $S_{1}$ at the intersection point of $E_{2}$ with the strict trasform of $C$; let $E_{1}$ be the exceptional divisor and $E_{2,1}$ the strict trasform of $E_{2}$. Denote by $\pi_{0}: X_{0} \rightarrow S_{0}$ the resolution of the induced fibration. The fiber over the general point of $E_{1}$ is a smooth elliptic curve.

If $K_{X_{0}}=\pi^{*}\left(K_{S_{0}}+\Delta_{0}\right)$ (this happens if $X_{0}$ is the resolution of a Weierstrass model $)$, then $K_{X_{j}}=\pi^{*}\left(K_{S_{j}}+\Delta_{j}\right) \forall j=1,2, \Lambda_{1}=\psi_{2}^{*}\left(\Lambda_{2}\right), \Lambda_{0}=\psi_{1}^{*}\left(\Lambda_{1}\right)-E_{1}$ and 
thus $\left(K_{S_{0}}+\Lambda_{0}\right) \cdot E_{2,1}=-1$ while $\left(K_{S_{0}}+\Lambda_{0}\right) \cdot E_{1}=0$.

Thus there exists a first log extremal contraction $\varphi_{1}$ contracting $E_{2,1}$ to a singular point and then a second $\log$ extremal contraction $\varphi_{2}$, which contracts $E$, the image of $E_{1}$, to the smooth point $p \in S . \varphi_{1} \cdot \varphi_{2}$ is a $\log$ extremal re-factorization of $\psi_{1} \cdot \psi_{2}$.

We will show that all the new contraction are of this type; in particular, we prove that if $E_{i}$ is contracted by $\varphi_{3}$, then $\left(K_{T}+\Delta_{T}\right) \cdot \hat{E}_{i}=0$, where $\hat{E}_{i}$ is the strict transform of $E_{i}$. In particular we will show:

Proposition 4.1. If a curve $E$ is log extremal on $T$, then $E$ passes through at most a singular point of $T$. In particular the contraction of $E$ improves the singularities and $\left(K_{S_{1}}+\Delta_{1}\right) \cdot \hat{E}=0$.

This property will allow us to re-factor $\varphi_{2} \cdot \varphi_{3}$, first contracting $\hat{E}_{i}$ to a smooth surface $S_{i_{1}}^{\prime}$ so that the previous algorithm (2.2 and 2.4) applies. In fact:

If $E$ is a log extremal curve on a smooth surface $V$ such that $(E)^{2}=-1$ and $\left(K_{V}+\Delta\right) \cdot E=0$, then

$$
\left(K_{V}+\Delta\right)=\psi^{*}\left(K_{S_{1}}+\Delta_{1}\right)
$$

where $\psi: S \rightarrow S_{1}$ is the contraction of $E$.

In particular if $\left(K_{S}+\Delta\right) \cdot C \in \mathbb{Z}$, for a 1-cycle $C$ on $S$, also $\left(K_{S_{1}}+\Delta_{1}\right) \cdot \psi_{*}(C) \in \mathbb{Z}$.

This process thus produces an iterative contraction algorithm; eventually $\phi$ is factored as:

$$
\varphi^{\prime}: S \rightarrow S^{\prime} \text { and } \varphi_{2}^{\prime}: S^{\prime} \rightarrow S_{2}^{\prime}=\bar{S}
$$

where $S^{\prime}$ is the smooth minimal resolution of $\bar{S}, \Sigma_{X / S^{\prime}}$ is not a divisor with simple normal crossings (if $S \neq S^{\prime}$ ) and $\varphi_{2}^{\prime}$ contracts only Hirzebruch-Jung strings.

Again most of the technical details are relegated in $\S 5$.

We start with the following:

Definition 4.2. Let $\mathcal{E}_{1}=\left\{E_{1_{1}}, \ldots E_{r_{1}, r_{1}-1}\right\} \ldots \mathcal{E}_{s}=\left\{E_{1_{s}}, \ldots E_{r_{s}, r_{s}-1}\right\}$ be $s$ disjoint Hirzebruch-Jung strings, as in (0.9), and $\mathrm{F}$ a smooth rational curve such that $E_{r_{j}-1, r_{j}} \cdot F=1, \forall j, 1 \leq j \leq s$.

Then $F$ is said to be at the feet of the strings $\mathcal{E}_{1} \cdots \mathcal{E}_{s}$.

By inspection on the graphs in Table 2, 3 and 4, and from general consideration, we deduce the following Remarks:

\section{Remarks 4.3.}

(1) If $E \notin \Sigma_{X / T}$, then $(\hat{E})^{2}=-1$ (0.14, part 2).

(2) If $E \in \Sigma_{X / T}$, then it is always at the "feet" except in the cases at the botton line of Table $3\left(I I^{*}, I_{0}^{*}\right)$, and a case in Table $4\left(I I^{*}, I V^{*}, I V\right)$.

(3) If E intersects only one exceptional graph at the "foot", then, by construction, $(\hat{E})^{2}=-1$. 
(4) If $(\hat{E})^{2}=-1$, and $\hat{E}$ intersects some chain of (-2) curves $\mathcal{E}$, then $\{\hat{E}, \mathcal{E}\}$ contracts to a smooth point.

(5) If $\hat{E}$ intersects 2 or more chains of (-2) curves, then $(\hat{E})^{2} \leq(-3)$.

(6) E cannot be at the "feet" of 4 or more exceptional curves and being exceptional on the minimal resolution ( $\bar{S}$ has at most quotient singularities).

In particular, we need to investigate the case of log extremal curves intersecting different chains. A crucial ingredient in the proofs is the following:

Lemma 5.6. Let $\left\{\mathcal{E}_{1}, \cdots, \mathcal{E}_{s}\right\}$ be $s=2,3$ disjoint Hirzebruch-Jung strings, and $F_{0}$ a smooth rational curve at the feet of $\left\{\mathcal{E}_{1}, \cdots, \mathcal{E}_{s}\right\}$.

Then the image of $F_{0}$ is not log extremal on $T$.

Now we consider a log extremal curve $E \notin \Sigma_{X / T}$.

Remark 4.4. Lemma 5.6 implies that if $\hat{E}$ intersect more than 1 Hirzebruch string, then $\hat{E}$ must also intersect a Hirzebruch-Jung string $\mathcal{E}$ of lenght $r \geq 2$; only the following cases can occur:

(A) $\hat{E}$ intersects at most 1 Hirzebruch-Jung string

(B) $\mathcal{E}$ is of type $\langle 5,2>$ and $\hat{E}$ intersects 1 other curve of self-intersection $\leq-3$

(C) $\mathcal{E}$ is of type $\langle 5,2>$ and $\hat{E}$ intersects 1 other curve $C$ of self-intersection -2

(D) $\mathcal{E}$ is of type $<5,3>$ and $\hat{E}$ intersects 1 other curve $C$ of self-intersection $\leq-3$

Proof of 4.1.

Case (1): $E \in \Sigma_{X / S}$. From Remark 4.4. and Lemma 5.5. follows that $\hat{E}$ has to be at the feet of only 1 string of $\log$ extremal curves or $\hat{E}$ has to be the curve of type $I I$ (as in 4.3, part 2).

In the first case the statement follows from 4.3 (part 3) and 5.10. For the second case, we obtain the same conclusion using Lemmas 5.7. and 5.9 and checking all the possible contractions.

Case (2) : $E \notin \Sigma$. Case by case checking (based on Remark 4.4 and applications of the lemmas 5.6 through 5.10$)$ shows that only case $(\mathrm{A})$ occurs and that $\left(K_{S_{1}}+\right.$ $\left.\Delta_{1}\right) \cdot \hat{E}=0$.

The following theorems are straightforward consequences of the above results. All the statements are obtained listing all the possible contractions for the curve not in $\Sigma_{X / T}$ or not at the "feet" and using the lemmas to show that only the one listed can occur.

Theorem 4.5. The graphs listed in Tables 2, 3, 4 are the only possible on the minimal resolution of $\bar{S}$. In particular only Hirzebruch-Jung strings can occur. The intersecting non log extremal curves need not to have simple normal crossings, but the sum of their coefficient has to equal the sum of the coefficient of the curves represented by empty circles in the Tables.

Proof. Set $\phi_{2} \cdot \phi_{1}=\phi_{2}^{\prime} \cdot \phi_{1}^{\prime}$, where $\phi_{1}^{\prime}$ denotes the contractions of $\hat{E}$ to a smooth point. Then the algorithm in the second part of Proposition 2.5. still applies (see note after 4.1 ). 


\section{Theorem 4.6.}

$$
\left(K_{S}+\Delta\right) \cdot \Delta=\left[\left(K_{\bar{S}}+\bar{\Delta}\right) \cdot \bar{\Delta}-\sum_{p \in U}\left(1-1 / n_{p}\right)\right]
$$

where $U=\{p / p$ is a singular points of $\bar{S}\}$ and the exceptional graph of $p$ is a Hirzebruch-Jung string of type $<n_{p}, q_{p}>$

Proof. From Proposition 4.1. we know that we can re-factor the contraction morphism, as decribed in $\S 2.1$. and that Proposition 2.2. still applies.

Proposition [G1]. Let $\pi: X \rightarrow S$ be an elliptic fibration between smooth varieties and $\Sigma$ a divisor of simple normal crossings. Let $n$ be the dimension of $X$. Then:

(1) $\chi\left(\mathcal{O}_{X}\right)=\chi\left(S, \mathcal{O}_{S}\left(-\pi_{*} K_{X / S}\right)\right)+\chi\left(S, \mathcal{O}_{S}\right)$

(2) If $n=3$ then, $\chi\left(\mathcal{O}_{X}\right)=-\frac{\left(K_{S}+\pi_{*} K_{X / S}\right) \cdot \pi_{*} K_{X / S}}{2}$.

Combining the above Proposition with Theorem 4.6 we have:

Corollary 4.7. Assume that all the exceptional curves for $\phi$ which are image of multiple fiber divisors have zero intersection with curves image of non multiple singular divisor. Then:

$$
\chi\left(\mathcal{O}_{X}\right)=-\frac{1}{2}\left\{\left[K_{\bar{S}}+\phi_{*} \pi_{*}\left(K_{X / S}\right)\right] \cdot \phi_{*} \pi_{*}\left(K_{X / S}\right)-\sum_{p \in V}\left(1-1 / n_{p}\right)\right\},
$$

where $V=\left\{p \in U\right.$ such that the fiber over the general point of $\phi^{-1}(p)$ is not multiple $\}$.

\section{$\S 5$ The Lemmas}

Preparation for the first step (Contractions to smooth points.)

The notation is as in (0.11): $\left\{E_{1}, \ldots, E_{k, k-1} \ldots E_{r, r-1}\right\}$ is a string of log extremal curves on a smooth surface $S$ and $\psi_{i}: S_{i-1} \rightarrow S_{i}, 1 \leq i \leq r$ the contraction of the log extremal curve $E_{i} \subset S_{i-1}$.

\section{Proposition 5.1.}

$$
\begin{aligned}
K_{S} \cdot E_{k, k-1} & =\psi_{1}^{*}\left(K_{1}\right) \cdot E_{k, k-1}=K_{1} \cdot E_{k, k-2}=\cdots=K_{k-1} E_{k}+\beta_{k-1}=\beta_{k} E_{k}^{2}+\beta_{k-1} \\
\Delta \cdot E_{k, k-1} & =\psi_{1}^{*}\left(\Delta_{1}\right) \cdot E_{k, k-1}=\Delta_{1} \cdot E_{k, k-2}=\cdots=\Delta_{k-1} E_{k}+\alpha_{k-1}=\alpha_{k} E_{k}^{2}+\alpha_{k-1} \\
-b_{k} & =\left(E_{k, k-1}\right)^{2}=\cdots=\left(E_{k, 2}\right)^{2}=\left(E_{k, 1}\right)^{2}
\end{aligned}
$$

Proof. It follows directly from the definitions.

\section{Lemma 5.2.}

(1) $\left(E_{k}\right)^{2}=-f_{k} / g_{k}$

(2) $\psi_{k-1}^{*}\left(E_{k}\right)=E_{k, 1}+\left(g_{k-1} / f_{k-1}\right) E_{k-1}$. 
Proof. $\psi_{k-1}^{*}\left(E_{k}\right)=E_{k, 1}+\mu_{k} E_{k-1}$, for some rational number $\mu_{k}$. Intersecting both sides of the equation first with $E_{k-1}$ and then with $E_{k, 1}$, we get $\mu_{k}=-1 /\left(E_{k-1}\right)^{2}$ and $\left(E_{k}\right)^{2}=-b_{k}-1 /\left(E_{k-1}\right)^{2}$.

Since $\left(E_{1}\right)^{2}=-b_{1}=-f_{1} / g_{1}$, (1) holds for $k=1$. Assuming by induction $\left(E_{k-1}\right)^{2}=-f_{k-1} / g_{k-1}$ we have $\left(E_{k}\right)^{2}=-\frac{f_{k}}{g_{k}}(0.4)$ and $\psi_{k-1}^{*}\left(E_{k}\right)=E_{k, 1}+$ $\frac{g_{k-1}}{f_{k-1}} E_{k-1}$.

\section{Lemma 5.3.}

(1) $\beta_{k}=\left(2-b_{k}+\beta_{k-1}\right) \frac{g_{k}}{f_{k}}, \forall k \geq 2$.

(2) $\beta_{k}=\left(g_{k}+1-f_{k}\right) / f_{k} \forall k$.

Proof. (1) Since all the curves in the string are rational,

$-2+b_{k}=K_{S} \cdot E_{k, k-1} \stackrel{(0.9)}{=} \beta_{k}\left(E_{k}\right)^{2}+\beta_{k-1}$.

Thus $\beta_{k}=\frac{2-b_{k}+\beta_{k-1}}{E_{k}^{2}}=\left(2-b_{k}+\beta_{k-1}\right) \frac{g_{k}}{f_{k}}$.

It follows also that $\beta_{1}=\left(2-b_{1}\right) / b_{1} \stackrel{(0.4)}{=}\left(g_{1}+1-f_{1}\right) / f_{1}$.

Thus (2) holds for $k=1$.

(2) Assume by induction $\beta_{k-1}=\left(g_{k-1}+1-f_{k-1}\right) / f_{k-1}$ and prove that the formula holds for $\beta_{k}$. Using part (1), we have:

$$
\begin{aligned}
& \beta_{k}=\left[2-b_{k}+\left(g_{k-1}+1-f_{k-1}\right) / f_{k-1}\right]\left(g_{k} / f_{k}\right) \\
& \beta_{k} \stackrel{(0.10)}{=}\left(2 g_{k}-b_{k} f_{k-1}+g_{k-1}+1-g_{k}\right) / f_{k} \\
& \beta_{k}=\left(g_{k}+1-f_{k}\right) / f_{k} \quad \square
\end{aligned}
$$

Preparation for the second step (Contractions of Hirzebruch-Jung strings.)

Now we consider a string of log extremal Hirzebruch-Jung curves $\left\{E_{1}, E_{2,1}, \ldots, E_{r, r-1}\right\}$ (as in 0.9).

\section{Remark 5.4:}

(1) Set $\Delta \cdot E_{k, k-1}=m_{k}$. Then $m_{k}$ is an integer (0.14, part 3), and

$$
m_{k}=\Delta_{1} \cdot E_{k, k-2}=\cdots=\Delta_{k-2} \cdot E_{k, 2}=\Delta_{k-1} \cdot E_{k, 1}+\alpha_{k-1}=\alpha_{k} \cdot\left(E_{k}\right)^{2}+\alpha_{k-1} .
$$

(2) $\alpha_{k}+\beta_{k}>0, \forall k$.

(3) Consequently, $0<\alpha<1$, since $\forall k \beta_{k} \leq 0$, and $\alpha_{k}<1$ (2.1.).

The goal of the first part of this session is to prove Proposition 2.2, which is part of the combinatorial data needed for the contraction algorithm.

We need the following lemma.

\section{Lemma 5.5.}

(1) $\alpha_{k}=1-g_{k} / f_{k}, \forall k$.

(2) $\alpha_{k}+\beta_{k}=1 / f_{k}$. 
Proof. (1) The crucial point in the proof of the Lemma is that $m_{k}$ is an integer (0.14, part 3).

We will show that the Lemma holds for $k=1$ and then use induction on $k$. Set $\alpha_{1}=\frac{p_{1}}{q_{1}}$, with $p_{1}, q_{1}$ relatively prime and

$$
0<p_{1}<q_{1}(5.4)
$$

then $m_{1}=-\alpha_{1} b_{1}=-p_{1} \frac{b_{1}}{q_{1}}$ (note that $\left.m_{1}<0\right)$.

Since $m_{1}$ must be an integer, $b_{1}=l q_{1}$, for some positive integer $l$; hence

$$
\alpha_{1}+\beta_{1}=\alpha_{1}+\frac{2-b_{1}}{b_{1}}>0 \text { and } p_{1} l+2-l q_{1}>0
$$

It follows that $l q_{1}-2<l p_{1}<l q_{1}$ and thus $l p_{1}=l q_{1}-1$.

Therefore :

$$
\left\{\begin{array} { r l } 
{ l } & { = 1 } \\
{ p _ { 1 } } & { = q _ { 1 } - 1 }
\end{array} \Rightarrow \left\{\begin{array}{l}
q_{1}=b_{1} \\
p_{1}=b_{1}-1
\end{array}\right.\right.
$$

Hence $\alpha_{1}=\left(b_{1}-1\right) / b_{1}=1-g_{1} / f_{1}$ and the lemma holds for $k=1$.

Assume by induction $\alpha_{k-1}=1-g_{k-1} / f_{k-1}$ and write $\alpha_{k}=p_{k} / q_{k}$, with $p_{k}, q_{k}$ relatively prime integers and $0<p_{k}<q_{k}$ (5.4).

By 5.4 and 5.2. $m_{k}=\alpha_{k}\left(E_{k}\right)^{2}+\alpha_{k-1}=-\alpha_{k} \frac{f_{k}}{g_{k}}+\alpha_{k-1}$. Then:

$$
\begin{aligned}
& m_{k}=-\left(1 / f_{k-1}\right)\left[\left(p_{k} / q_{k}\right)\left(b_{k} f_{k-1}-g_{k-1}\right)+g_{k-1}\right]+1 \\
& m_{k}=-\left(1 / f_{k-1}\right)\left[\left(p_{k} / q_{k}\right) f_{k}+g_{k-1}\right]+1
\end{aligned}
$$

$m_{k}$ must be an integer, and thus $f_{k}=l_{k} q_{k}$ for some positive integer $l_{k} ; 5.5 .1$ becomes then:

$$
m_{k}=-\frac{1}{f_{k-1}}\left(p_{k} l_{k}+g_{k-1}\right)+1
$$

By $(5.3)$

$$
\alpha_{k}+\beta_{k}>0 \Longleftrightarrow \alpha_{k} f_{k}+\left(2-b_{k}\right) g_{k}+\left(g_{k-1}+1-f_{k-1}\right)>0, \forall k \geq 2
$$

that is

$$
p_{k} l_{k}+\left(2-b_{k}\right) g_{k}+\left(g_{k-1}+1-f_{k-1}\right)>0 .
$$

By (0.10) this last equation becomes

$$
p_{k} l_{k}>b_{k} f_{k-1}-f_{k-1}-1-g_{k-1}
$$

also $l_{k} p_{k}<f_{k} \stackrel{(5.4)}{=} b_{k-1} f_{k-1}-g_{k-1}$. 
Combining the last two inequalities we get:

$$
\begin{gathered}
\left(b_{k}-1\right) f_{k-1}-1<p_{k} l_{k}+g_{k-1}<b_{k} f_{k-1} \quad \text { i.e. } \\
\left(b_{k}-1\right) f_{k-1} \leq p_{k} l_{k}+g_{k-1}<b_{k} f_{k-1} .
\end{gathered}
$$

Since $m_{k}$ is an integer, $f_{k-1}$ must divide $p_{k} l_{k}+g_{k-1}$ (5.5.2); thus: $p_{k} l_{k}=\left(b_{k}-\right.$ 1) $f_{k-1}-g_{k-1}$, that is

$$
p_{k} l_{k}=f_{k}-f_{k-1}
$$

Dividing both side of (5.5.3) by $q_{k} l_{k}=f_{k}$, we have:

$$
\begin{aligned}
\alpha_{k} & =\frac{p_{k} l_{k}}{q_{k} l_{k}}=1-\frac{f_{k-1}}{f_{k}} \text { or } \\
\alpha_{k} & =1-g_{k} / f_{k} .
\end{aligned}
$$

(2) $\alpha_{k}+\beta_{k}=1 / f_{k}$ follows from part (1), 5.3. and (0.10).

\section{Preparation for the third step}

First the consider the case of a log extremal curve at the feet of $s$ Hirzebruch-Jung strings $\left\{\mathcal{E}_{1}, \cdots \mathcal{E}_{s}\right\}, s=2,3$ (see 4.3 ).

Lemma 5.6. Let $\left\{\mathcal{E}_{1}, \cdots, \mathcal{E}_{s}\right\}$ be $s=2,3$ disjoint Hirzebruch-Jung strings, and $F_{0}$ a smooth rational curve at the feet of $\left\{\mathcal{E}_{1}, \cdots, \mathcal{E}_{s}\right\}$.

Then the image of $F_{0}$ is not log extremal on $T$.

Proof. We will prove the statement for the case $s=2$. The same argument works for the case $s=3$, but the notation is more complicated.

Assume that such a $F_{0}$ exists and derive a contradiction. The proof is along the same line of 5.5. $\left(\Delta \cdot F_{0}\right.$ is an integer and the contraction of the image of $F_{0}$ is log extremal.)

Let $\psi_{1}: S \rightarrow T_{1}$ be the contraction of the string $\mathcal{E}_{1}$ and $\psi_{2}: T_{1} \rightarrow T_{2}$ the contraction of the string $\mathcal{E}_{2}$. Set $F_{j}=\left(\psi_{j}\right)_{*}\left(F_{j-1}\right)$ and denote by $\psi_{3}: T_{2} \rightarrow T_{3}$ the contraction of $F_{2}$; set $K_{T_{2}}=\psi_{3}^{*}\left(K_{T_{3}}\right)+\zeta F_{2}$ and $\Delta_{T_{2}}=\psi_{3}^{*}\left(\Delta_{T_{3}}\right)+\eta F_{2}$.

Then

$$
\left(K_{S}+\Delta\right) \cdot F_{0}=(\eta+\zeta)\left(F_{2}\right)^{2}+\frac{1}{f_{r_{1}}}+\frac{1}{f_{r_{2}}} .
$$

If we set $\left(F_{0}\right)^{2}=-b$, it is easy to see that

$$
\left\{\begin{aligned}
\left(F_{2}\right)^{2} & =-b+\frac{g_{r_{1}}}{f_{r_{1}}}+\frac{g_{r_{2}}}{f_{r_{2}}} \\
\zeta & =\left[b-2-\left(\frac{g_{r_{1}}-f_{r_{1}}+1}{f_{r_{1}}}+\frac{g_{r_{2}}-f_{r_{2}}+1}{f_{r_{2}}}\right)\right] /\left(\frac{g_{r_{1}}}{f_{r_{1}}}+\frac{g_{r_{2}}}{f_{r_{2}}-b}\right)
\end{aligned}\right.
$$


That is $\zeta=-1+\frac{f_{r_{1}}+f_{r_{2}}}{b_{1} f_{r_{1}} f_{r_{2}}-f_{r_{2}} g_{r_{1}}-f_{r_{1}} g_{r_{2}}}$. Set $\eta=p / q$. Since $\Delta \cdot F_{0} \in \mathbb{Z}$,

$$
\frac{1}{\left(f_{r_{1}} f_{r_{2}}\right)}\left\{\frac{p}{q}\left(b f_{r_{1}} f_{r_{2}}-g_{r_{2}} f_{r_{1}}-g_{r_{1}} f_{r_{2}}\right)+g_{r_{1}} f_{r_{2}}+g_{r_{2}} f_{r_{1}}\right\}
$$

must be an integer. Therefore $b f_{r_{1}} f_{r_{2}}-g_{r_{2}} f_{r_{1}}-g_{r_{1}} f_{r_{2}}=A q$, for some integer $A$; thus $\zeta=\frac{-A q+f_{r_{1}}+f_{r_{2}}}{A q}$ and $\frac{1}{\left(f_{r_{1}} f_{r_{2}}\right)}\left\{\frac{p}{A}+g_{r_{1}} f_{r_{2}}+g_{r_{2}} f_{r_{1}}\right\}$ is an integer.

The singularities are log terminal $(\zeta>-1)$, thus $A q$ is a positive integer. As in the proof on 5.5 have $-\zeta<\eta<1$ and thus: $-\zeta q A<p A<q A$, that is

$$
\begin{gathered}
b f_{r_{1}} f_{r_{2}}-\left(f_{r_{1}}+f_{r_{2}}\right)-f_{r_{1}} g_{r_{2}}-f_{r_{2}} g_{r_{1}}<p A<p\left(b f_{r_{1}} f_{r_{2}}-f_{r_{2}} g_{r_{1}}-f_{r_{1}} g_{r_{2}}\right) \\
b f_{r_{1}} f_{r_{2}}-\left(f_{r_{1}}+f_{r_{2}}\right)<A p+f_{r_{2}} g_{r_{1}}+f_{r_{1}} g_{r_{2}}<b f_{r_{1}} f_{r_{2}}
\end{gathered}
$$

Since $\Delta \cdot F_{0}$ is an integer, $f_{r_{1}} f_{r_{2}}$ must divide $A p+f_{r_{2}} g_{r_{1}}+f_{r_{1}} g_{r_{2}}$, and thus such $p$ and $q$ exist if and only if $f_{r_{1}}+f_{r_{2}}>f_{1} f_{r_{2}}$. This impossible because $\mathcal{E}_{1}$ and $\mathcal{E}_{2}$ are Hirzebruch-Jung strings.

Thus a log extremal curve $E$ can never be at the "feet" of 2 or more exceptional curves (see also 4.3).

Here starts a series Lemmas and Corollaries which will be used to prove that all the log extremal curves contracted by $\varphi_{3}$ (see $\S 2$ ) have self-intersection -1 on the minimal resolution. Again the notation is as in (0.9).

Lemma 5.7. Let $\left\{E_{1}, \ldots, E_{r-1, r}\right\}$ be a Hirzebruch-Jung string and $\psi: S \rightarrow S_{r}$ the corresponding log extremal contraction. Let $F_{0}$ be a curve on $S$ such that $E_{1} \cdot F_{0}=1$. Set $F_{r}=(\psi)_{*}\left(F_{0}\right)$. Then:

$$
\left(K_{S}+\Delta\right) \cdot F_{0}=\left(K_{S_{r}}+\Delta_{r}\right) \cdot F_{r}+\frac{1}{f_{1}}+\sum_{k=1}^{r-1} \frac{1}{f_{k} f_{k+1}} .
$$

Proof. Let $\psi_{j}: S_{j-1} \rightarrow S_{j}$ be as in (0.9.) and set $F_{j}=\left(\psi_{j}\right)_{*}\left(F_{j-1}\right)$. The statement follows from

$$
\left(K_{S}+\Delta\right) \cdot F_{0}=K_{S_{r}}+\Delta_{r}+\left(\alpha_{1}+\beta_{1}+\cdots+\left(\alpha_{k}+\beta_{k}\right) E_{k} \cdot F_{k-1}+\ldots\left(\alpha_{r}+\beta_{r}\right) E_{r} \cdot F_{r-1}\right.
$$

and Proposition 5.2.

Corollary 5.8. In the hypothesis and notation of Lemma 5.7., let $r=2,3$. Then $\left(K_{S}+\Delta\right) \cdot F_{0}=0$.

Proof. By 5.7,

$$
0>\left(K_{S}+\Delta\right) \cdot F_{0}=\left(K_{S_{r}}+\Delta_{r}\right) \cdot F_{r}+\frac{1}{f_{1}}+\sum_{k}^{r-1} \frac{1}{f_{k} f_{k+1}} \text {. }
$$


(1) $\mathrm{r}=2$. It's enough to observe :

$$
1 / f_{1}+1 /\left(f_{1} f_{2}\right)=1 / b_{1}+1 / b_{1}\left(b_{1} b_{2}-1\right)=b_{2} /\left(b_{1} b_{2}-1\right)<1 .
$$

(In fact, $b_{2}<\left(b_{1} b_{2}-1\right)$ unless $b_{1}=1$; note also that $\left(K_{S}+\Delta\right) \cdot F_{0}$ is a positive integer.)

(2) $\mathrm{r}=4$. As above we have :

$$
1 / f_{1}+1 /\left(f_{1} f_{2}\right)+1 /\left(f_{2} f_{3}\right)=\left[b_{2} b_{3}-1\right] /\left[b_{3}\left(b_{1} b_{2}-1\right)-b_{1}\right]<1
$$

Lemma 5.9. Let $\left\{E_{1}, E_{1,2}, E_{2,3}\right\}$ be a Hirzebruch-Jung string and $\psi: S \rightarrow$ $S_{3}$ the corresponding log extremal contraction. Let $F_{0}$ be a curve on $S$ such that $E_{1,2} \cdot F_{0}=1$ and set: $\psi_{*}\left(F_{0}\right)=F_{3}$. Then:

$$
\left(K_{S}+\Delta\right) \cdot F_{0}=\left(K_{S_{3}}+\Delta_{3}\right) \cdot F_{3}+\frac{1}{f_{2}}+\frac{1}{f_{3}}+\frac{f_{1}}{f_{2}}
$$

Proof. Procede as in the proof of Lemma 5.7.

The following proposition shows that the strict transform on $S_{1}$ of any log extremal curve contracted by $\varphi_{3}$ has intersection number 0 with $K_{S_{1}}+\Delta_{1}$.

Lemma 5.10. Let $\left\{E_{1} \ldots E_{r-1, r}\right\}$ be as in 2.2. Assume that $E$ is a log extremal curve on $S_{r}$ and denote by $\hat{E}$ the strict transform of $E$. Then if $\hat{E} \cdot E_{r-1, r}=1$, we have:

$$
\left(K_{S}+\Delta\right) \cdot \hat{E}=0
$$

Proof. Without loss of generality (2.2.), we can assume that $\hat{E}^{2}=-1$ and $\left(K_{S}+\right.$ $\Delta) \cdot \hat{E} \geq 0$. Since

$$
\left(K_{S}+\Delta\right) \cdot \hat{E}=\left(K_{S_{r}}+\Delta_{r}\right) E+\alpha_{r}+\beta_{r}
$$

we have:

$$
0>\left(K_{S_{r}}+\Delta_{r}\right) E=\left(K_{S}+\Delta\right) \cdot \hat{E}-1 / f_{r} ;
$$

Now $\left(K_{S}+\Delta\right) \cdot E$ has to be zero, because $1 / f_{r}<1$ and $\left(K_{S}+\Delta\right) \cdot E \in \mathbb{Z}$.

\section{REFERENCES}

[BrE. Brieskorn, Rationale singularitaten komplexen Flächen, Invent. Math. 4 (1968), 336-358.

[G1A. Grassi, On minimal model of elliptic threefolds., Math. Ann. 290 (1991), $287-301$. 
[HaA] Hartshorne, Ample vector bundles, Publ. Math. IHES 29 (1966), 63-94.

[Ha2]. Hartshorne, Algebraic Geometry, Springer-Verlag, New York, 1977.

[KaYl] Kawamata, Characterization of Abelian varieties., Compositio Math. 43 (1981), 253-276.

[Kaฆ] Kawamata, Kodaira dimension of certain algebraic fiber spaces, J. Fac. Sci. Tokyo Univ. IA 30 (1983), 1-24.

[Ka8] Kawamata, Hodge theory and Kodaira dimension., Algebraic Varieties and Analytic Varities, (S. Iitaka, editor) Adv. Stud. Pure Math. 1, Kinokuniya, Tokyo, 1983, pp. 317-327.

[Ka4] Kawamata, The cone of curves of algebraic varieties, Ann. of Math. 119 (1984), 603-633.

[Kab] Kawamata, Pluricanonical systems on minimal algebraic varieties, Invent. Math. 79 (1985), 603-633.

[Ka6]. Kawamata, Minimal models and the Kodaira dimension of algebraic fiber spaces., J. Reine Angew. Math 363 (1985), 1-46.

[KdK. Kodaira, On compact analytic surfaces II and III, Annals of Math 77,78 (1963), 563-626, 1-40.

[Ko]. Kollár, Flops, Nagoya Math. J. 113 (1989), 15-36,.

[KMMҢawamata, K. Matsuda, K.Matsuki, Introduction to the minimal model problem, Proc. Sym. Alg. Geom. Sendai 1985, Adv. Stud. Pure Math. 10, Kinokuniya, Tokyo, 1985, pp. 283-360.

$[\mathbf{K z N}$. Katz, The regularity theorem in algebraic geometry, Proc. Internat. Congr. Math., Nice, Gauthier Villars 1 (1970), 437-443.

[MiY. Miyaoka, On the Kodaira dimension of minimal threefolds, Math. Ann 281 (1988), 325-332.

[Mळ]. Mori, Flip theorem and the existence of minimal models for 3-folds, Jour. Amer. Math. Soc. 1 (1988), 567-588.

[SaF. Sakai, Classification of normal surfaces, Algebraic Geometry Bowdoin, Proc. Sympos. Pure Math. 46, 1, Amer. Math. Soc., Providence, 1987, pp. 451-465.

$[\mathbf{Z a}$ O. Zariski, The theorem of Riemann-Roch for high multiples of an effective divisor on an algebraic surface, Ann. of Math. 76 (1962), 560-615.

Department of Mathematics, Tufts University, Medford, MA 02155

E-mail address: agrassi@jade.tufts.edu 